\section{PCAOs: uncommon antisense}

\section{By Michael J. Haas, Senior Writer}

Transcription factors and other molecules involved in interactions inside cell nuclei are important targets for treating many diseases, but a number of characteristics have made them difficult drug targets. A research team from Memorial Sloan-Kettering Cancer Center has proposed a solution to the problem: peptide-conjugated oligonucleotides.

Targets located inside cell nuclei are not druggable with conventional antibodies, and their large binding sites-typical for protein-protein and protein-DNA interactions-make them difficult to target with small molecules. Conventional inhibitors of intranuclear interactions-peptidomimetics or oligonucleotides-often lack specificity for a given cell type and may metabolize relatively quickly in vivo, reducing their effectiveness as drugs.

A team led by Robert Benezra reported in Nature Biotechnology the design of a conjugated molecule that specifically inhibited a key tumor transcription factor to suppress tumor growth in mice. ${ }^{1}$ Besides yielding a viable compound for development, Benezra told SciBX that the results provide proof of concept for a method of developing other selective, metabolically stable inhibitors of intranuclear interactions in cancer and other diseases.

The approach produces a peptide-conjugated antisense oligonucleotide (PCAO) by linking a conventional antisense oligo (AO) 'payload' to a peptide fragment 'warhead' that homes only to the desired cell type. Chemical modifications to the peptide fragment can increase its resistance to metabolism-and therefore increase its in vivo half-life-and the choice of peptide fragment ensures selective uptake by cells.

In the Nature Biotechnology paper, Benezra's team described the design of a PCAO that inhibited a transcription factor whose functions include regulation of angiogenesis in tumors, inhibitor of DNA binding-1 (Id1). Besides its angiogenic role, Id 1 was chosen because it presented a challenging test case for selectivity: it is structurally similar to the proteins that it binds.

For their warhead, Benezra's team chose $\mathrm{F} 3$, a peptide fragment that homes to nucleolin expressed on tumor endothelial cells. Nucleolin is a cell-surface receptor that is expressed on many cell types, but-for unknown reasons-F3 only binds nucleolin expressed on tumor endothelia, Benezra said.

The molecule's payload was a known AO inhibitor of Id1.
In vitro studies demonstrated that the Id1-PCAO was taken up only by tumor-but not normal-endothelial cells, where it downregulated expression of Id1. In allograft mice, Id1-PCAO suppressed primary tumor growth.

"Weve seen effectiveness in primary and metastatic tumor growth," Benezra told SciBX.

"Right now, we don't know whether we can get into other cell types," he added. "But all known tumor blood vessels express Id1, so the effects of Id1-PCAO on endothelial cells and blood vessels should be generalizable and allow us to go after a whole range of cancers."

Going forward, Benezra said, "We would develop Id1-PCAO for use alone or in combination with chemotherapy. Chemotherapy induces the mobilization of Id1-requiring endothelial progenitors from the bone marrow. We think this is responsible, in part, for tumor resistance to chemotherapy and our Id1-PCAO should effectively target those cells."

Benezra said his team also is developing PCAOs to target tumor proliferation for use in combination with Id1-PCAO.

Memorial Sloan-Kettering obtained a provisional patent on the work of Benezra's team and is negotiating licensing rights to develop Id1-PCAO with AngioGenex Inc., which would complete preclinical development and begin clinical trials.

"We expect to file an IND within 15 months," company COO William Garland told SciBX.

There are at least a dozen companies working in the antisense space for cancer and other indications. At least one of them, AVI BioPharma Inc., says it has developed an antisense approach that is similar to PCAO technology and has established an advanced position with it.

Bob Brown, VP of research and technology at the antisense company Genta Inc., told SciBX that the PCAO technology constituted an interesting addition to the repertoire of AOs.

"The demonstration in the paper is quite elegant," Brown said. "The technology should be extendable to any disease where you want to target neovasculature" and specifically "to cells that overexpress or inappropriately express nucleolin on their surfaces."

But Brown would want to see more data before deciding that Id1PCAO had the specificity the researchers claimed. "Those claims would need to be supported by full histopathological analysis in a preclinical toxicology study," he said.

Supplementary figures 6 and 7 to the Nature Biotechnology paper show some preliminary data for biodistribution of Id1-PCAO-in five tissues, four hours after administration-but Brown said these "do not constitute a histopathological analysis of the tissues."

"One needs more than one time point and more than a single administration of the compound" to compare biodistribution of PCAOs to conventional AOs, he said. "Repeated administration often reveals tissue accumulation that doesn't show up after only one administration. The ideal comparison would be both types of oligonucleotides administered via a pump for 20 days, then a full histopath analysis of the animals, 
including isolation and homogenization of samples in each tissue and a high-sensitivity assay for the presence of the oligonucleotide."

Brown added: "In particular, I'd like to see a comparative analysis of at least kidney, liver, spleen, marrow, lung and colon [tissue]. The colon seems to be a target in some cases only after repeated administration."

In some in vivo studies reported by Benezra's team, Id1-PCAO was administered by subcutaneous pumps implanted in the mice, to deliver a continuous dose of the compound over a 20-day period.

That mode of delivery is not going to be used in clinical trials, Garland said. "Initial administration is likely to be short-term parenteral infusion over about 90 minutes," he told SciBX. "If we observed no acute infusion-associated events in Phase I, the infusion time would be decreased to 30 minutes for Phase IIa patients."

Genta's Genasense IV oblimersen sodium, an antisense agent targeting Bcl-2 mRNA, is in development as an injectable for use in conjunction with chemotherapy. The compound is in a second Phase III trial for advanced melanoma and completed a Phase III trial for chronic lymphocytic leukemia in 2007.

In December 2006, the FDA issued a not-approvable letter for Genasense plus chemotherapy to treat relapsed or refractory chronic lymphocytic leukemia. The company appealed this decision twice in 2007. ${ }^{2}$ On Jan. 14 this year, the FDA announced it had extended its review of the appeal; Genta now expects a decision in 1Q08.

Reata Pharmaceuticals Inc. is "pleased that transcriptional regulation is being looked at more broadly," Melissa Krauth, VP of corporate and commercial development, told SciBX. "It has great therapeutic potential."

"We're very interested in modulating transcription factors in the cancer setting," said Chris Wigley, VP of research at Reata. "This [PCAO technology] would seem to work well for that, so it is encouraging in that regard. My understanding of the antisense landscape is that this represents a stride forward in terms of targeting a specific cell type."

Reata has RTA402, a synthetic terpinoid that inhibits the transcription factor NF- $\kappa B$ and the signal transducer and activator of transcription 3 (STAT3), in a Phase II trial for cancer. The company expects to complete that trial in 12-18 months and to begin Phase II trials of RTA402 for renal cancer and rheumatoid arthritis within the next 9 months.

As to PCAOs, "I'm encouraged by the data and proof of concept," Wigley said. "I could see how, in some situations, PCAOs could modulate transcription factors in specific cell types, in certain instances outside of cancer."

But Reata isn't interested in incorporating PCAO technology into its own programs. "We don't see that it would be necessary to use such a complex technology to get into the nucleus if you can affect signaling pathways in the cytoplasm," said Krauth.

Colin Meyer, VP of product development at Reata, doesn't think PCAO technology is quite ready for commercial application. "The technology is promising, but needs more benchmarking data," he said. "For instance, the dosing regimen is cumbersome and the given data make it hard to assess the impact Id1-PCAO would have against large or existing tumors."
Reata executives also expressed concerns about the limited toxicity data supplied in the paper.

"There are general safety issues for antiangiogenics that will have to be addressed," Meyer said, adding that he would like to see comparisons to other antiangiogenics in treatment models and in models of VEGF inhibitor resistance.

Though the PCAO approach has new aspects, Patrick Iversen, SVP of $\mathrm{R} \& \mathrm{D}$ at AVI BioPharma, described the work of Benezra's team as "very similar to ongoing efforts at AVI."

"The advantages of conjugating a delivery peptide to an antisense oligomer should provide for a significant decrease in the dose required to produce therapeutic benefit, due to the targeting character of the peptide," Iversen told SciBX. "I believe AVI has taken this sort of idea beyond the work described in [the paper] so that now we can deliver to multiple selected organs."

Iversen added: "We have described how to make efficient peptideconjugated oligomers a great deal, and successfully used peptide-conjugated antisense agents in our in vivo antiviral studies, antibacterial studies and for the delivery of splice-altering agents to muscle-including heart muscle-for the treatment of Duchenne muscular dystrophy."

AVI has two of its NeuGene antisense oligomers in clinical trials. AVI-4658 is in a Phase I/II trial for Duchenne muscular dystrophy; the company has not disclosed the compound's mechanism of action. AVI-5126, which targets c-myc mRNA, is in a Phase II trial for ex vivo treatment of re-stenosis in coronary artery bypass graft surgery, with trial completion expected in late 2008. AVI also expects to start a Phase I/II trial of AVI-5126 for re-stenosis following angioplasty in early 2008.

AVI's antisense technology uses phosphorodiamidate morpholino oligos (PMOs), which are similar in function to oligonucleotides but have chemical structures that make them unrecognizable to cellular proteins. Thus PMOs are not susceptible to normal intracellular degradation, nor are they likely to activate intracellular molecules such as NF- $\kappa \mathrm{B}$.

PMOs are linked to cell-penetrating peptides that allow them to enter cells.

"PMOs are likely to be the optimal platform for peptide-conjugated antisense agents," Iversen said, "because there is no net molecular charge to the PMO platform."

By contrast, the phosphate-bearing oligonucleotide moiety of Id1PCAO has a net negative charge-and Benezra told SciBX this has caused the compound to accumulate in the kidneys.

"It's a concern because we think we're losing a lot of the PCAO to this accumulation and subsequent excretion," Benezra said, "but we see no pathological effects of this." The team is looking for ways to reduce excretion and thereby lower the threshold for an effective dose.

He also said that the results obtained for Id1-PCAO thus far did not point to a need for additional toxicity analyses.

"While we haven't done the precise histological experiment [that Bob Brown described], weve done continuous infusions of Id1-PCAO over 20 days with no observable toxicity, which implies that the possibility of toxic accumulation in vital organs seems very remote," he said.

He said that it was also unlikely that Id1-PCAO would cause the same toxic side effects as do VEGF inhibitors. "Id1 is not expressed in 
normal vasculature," he said. "It is more specifically expressed in the tumor endothelium than VEGF receptor," so targeting Id1 should incur less risk of toxicity, he said.

Benezra emphasized that his team has not even found the maximum tolerated dose of Id1-PCAO.

After it has in-licensed the compound, AngioGenex plans to conduct pharmacological studies to identify which cancers respond best to Id1-PCAO, but Garland and Benezra agreed that so far the breast cancer model has been the most successful.

Garland noted that metastasis of breast cancer to the brain is a major concern. For this reason, Benezra's team is seeking to develop PCAOs that will cross the blood-brain barrier to target gliomal tumors.

Benezra told SciBX that PCAO technology could be used to target other transcription factors, transcription factor activators, DNA and RNA for cancer and other diseases. But he added: "We feel the best targets at this point are ones in which partial reduction [of expression] is shown to have a physiological effect," such as tumor reduction or suppression.

\section{REFERENCES}

1. Henke, E. et al. Nat. Biotechnol.; published online Jan. 6, 2008; doi:10.1038/nbt1366

Contact: Robert Benezra, Department of Cancer Biology and Genetics, Memorial Sloan-Kettering Cancer Center, New York, N.Y. e-mail: r-benezra@ski.mskcc.org

2. Mikulski, M. BioCentury 15(37), A6; Aug. 20, 2007

COMPANIES AND INSTITUTIONS MENTIONED

AngioGenex Inc. (OTCBB:AGGX), New York, N.Y.

AVI BioPharma Inc. (NASDAQ:AVII), Portland, Ore.

Genta Inc. (NASDAQ:GNTA), Berkeley Heights, N.J.

Memorial Sloan-Kettering Cancer Center, New York, N.Y.

Reata Pharmaceuticals Inc., Irving, Texas 